\title{
Case Finding and Medical Treatment of Type 2 Diabetes among Different Ethnic Minority Groups: The HELIUS Study
}

\author{
Marieke B. Snijder, ${ }^{1}$ Charles Agyemang, ${ }^{1}$ Ron J. Peters, ${ }^{2}$ Karien Stronks, ${ }^{1}$ \\ Joanna K. Ujcic-Voortman, ${ }^{3}$ and Irene G. M. van Valkengoed ${ }^{1}$ \\ ${ }^{1}$ Department of Public Health, Academic Medical Center, Amsterdam, Netherlands \\ ${ }^{2}$ Department of Cardiology, Academic Medical Center, Amsterdam, Netherlands \\ ${ }^{3}$ Public Health Service of Amsterdam, Amsterdam, Netherlands \\ Correspondence should be addressed to Marieke B. Snijder; m.b.snijder@amc.uva.nl
}

Received 30 August 2016; Accepted 13 December 2016; Published 5 January 2017

Academic Editor: Ulrike Rothe

Copyright (C) 2017 Marieke B. Snijder et al. This is an open access article distributed under the Creative Commons Attribution License, which permits unrestricted use, distribution, and reproduction in any medium, provided the original work is properly cited.

\begin{abstract}
Aims. Prevention of diabetes complications depends on the level of case finding and successful treatment of diabetes, which may differ between ethnicities. Therefore, we studied the prevalence by age, awareness, treatment, and control of type 2 diabetes, among a multiethnic population. Methods. We included 4,541 Dutch, 3,032 South-Asian Surinamese, 4,109 African Surinamese, 2,323 Ghanaian, 3,591 Turkish, and 3,887 Moroccan participants (aged 18-70 y) from the HELIUS study. The prevalence of diabetes was analysed by sex, ethnicity, and 10-year age groups. Ethnic differences in the prevalence, awareness, treatment, and control of diabetes were studied by logistic regression. Results. From the age of 31-40 years and older, the prevalence of diabetes was 3 to 12 times higher among ethnic minority groups than that among the Dutch host population. Awareness and medical treatment of diabetes were 2 to 5 times higher among ethnic minorities than that among Dutch. Among those medically treated, only $37-53 \%$ had HbAlc levels on target $(\leq 7.0 \%)$; only Dutch men had HbAlc levels on target more often $(67 \%)$. Conclusions. Our results suggest that the age limit for case finding among ethnic minority groups should be lower than that for the general population. Importantly, despite higher awareness and treatment among ethnic minorities, glycemic control was low, suggesting a need for increased efforts to improve the effectiveness of treatment in these groups.
\end{abstract}

\section{Introduction}

People with diabetes are at increased risk for cardiovascular disease and its related complications. Glycemic control may reduce this risk [1-4], but this largely depends on the level of case finding and adequate care. In the USA, the awareness, treatment, and control of diabetes have been shown to increase over the last decades, suggesting overall improvements in case finding and health care $[5,6]$. However, ethnic differences in awareness and glycemic control remained, with lower awareness among Hispanic and Asian participants compared with African Americans and White Americans and less glycemic control among African Americans and Hispanic Americans, as compared with White Americans [5-7].
In Europe, a higher prevalence of diabetes is found among ethnic minority groups, such as African, Turkish, Moroccan, and particularly South-Asian origin groups, as compared with European host populations [8-13]. Data on awareness, treatment, and control among these groups, however, are very limited and the results are inconsistent. In contrast to the USA, the awareness seems to be higher among African and South-Asian ethnic minority groups as compared with the European host population [14-16]. For example, in 2003, 78\% of South-Asians and Africans living in the Netherlands were aware of their diabetes, as compared with 58\% among Dutch population [15]. Consistent with results from the USA, however, studies from the UK have also shown poorer diabetes control among African and South-Asian ethnic minority 
groups [17-19]. Information on awareness, treatment, and glycemic control among other large ethnic minority groups, such as Turkish and Moroccan groups, is currently lacking.

Diabetes appears to develop at a younger age in some ethnic minority groups, such as South-Asians, Africans, and Turkish and Moroccan origin groups, as compared to the host population $[11,15,17,19]$. This may have consequences for the age at which case finding for diabetes should start among some ethnic groups. If the prevalence of diabetes is already substantially higher at a younger age among ethnic minority groups, case finding should also start at younger age. However, most previous studies had a limited age range or numbers were too small to adequately stratify by age.

Therefore, the aim of this study was to gain insight into both the age-specific prevalence of diabetes and the current levels of awareness, medical treatment, and glycemic control, among different ethnic groups. Results of this study may facilitate case finding and help the development of better guidelines for prevention and health care of diabetes.

\section{Materials and Methods}

2.1. Study Population. The HEalthy LIfe in an Urban Setting (HELIUS) study is a multiethnic cohort study conducted in Amsterdam which has been described in detail elsewhere [20]. In brief, baseline data collection took place in 20112015 and included people aged 18 to 70 years from six ethnic groups living in Amsterdam, that is, those of Dutch, SouthAsian Surinamese, African Surinamese, Ghanaian, Moroccan, and Turkish origin. Participants were randomly, stratified by ethnicity, sampled from the municipal register. Data were collected by questionnaire and a physical examination in which biological samples were also obtained. The HELIUS study is conducted in accordance with the Declaration of Helsinki and has been approved by the AMC Ethical Review Board. All participants provided written informed consent.

A flowchart of the recruitment for the HELIUS study is given in Figure 1. A total of 90,019 subjects were sent an invitation letter (and a reminder after 2 weeks) by mail. We were able to get a response from $55 \%$ either by response card or after a home visit by an ethnically matched interviewer. Of those, 24,789 agreed to participate (participation rate of $45 \%$ ). After a positive response, participants received a confirmation letter of the appointment for the physical examination, including a digital or paper version of the questionnaire (depending on the preference of the subject). Participants who were unable to complete the questionnaire themselves were offered assistance from a trained ethnically matched interviewer.

For the current study, we used baseline data of all participants in whom questionnaire data as well as data from the physical examination were available $(n=22,165)$. We excluded those of Javanese Surinamese $(n=233)$ or unknown Surinamese $(n=267)$ origin due to small numbers and we also excluded those with another/unknown ethnic origin $(n=48)$. Participants who had missing data on the presence of diabetes $(n=113)$ were also excluded. Finally, participants who reported an age of onset of their diabetes before the age of 30 years and to have started insulin injections immediately after being diagnosed $(n=21)$ were also excluded, because these participants are very likely to have type 1 diabetes. Therefore, data of 21,483 participants were available for analyses, including 4,541 Dutch, 3,032 South-Asian Surinamese, 4,109 African Surinamese, 2,323 Ghanaian, 3,591 Turkish, and 3,887 Moroccan origin participants.

2.2. Ethnicity. Ethnicity was defined according to the country of birth of the participant as well as that of his/her parents, which is currently the most widely accepted and most valid assessment of ethnicity in Netherlands [21]. Specifically, a participant is considered to be of non-Dutch ethnic origin if he/she fulfils either of the following criteria: (1) he or she was born abroad and has at least one parent born abroad (first generation) or (2) he or she was born in Netherlands but both his/her parents were born abroad (second generation). Of the Surinamese immigrants in Netherlands, approximately $80 \%$ are of either African or South-Asian origin. Surinamese subgroups were classified according to self-reported ethnic origin. Participants were considered to be of Dutch origin if the person and both parents were born in Netherlands.

2.3. Diabetes. Fasting blood samples were used to determine the concentration of glucose by spectrophotometry, using hexokinase as primary enzyme (Roche Diagnostics, Japan). Participants were asked to bring their prescribed medications, which were coded according to the Anatomical Therapeutic Chemical (ATC) classification [22]. Diabetes mellitus was considered to be present if the participants' fasting glucose level was $\geq 7.0 \mathrm{mmol} / \mathrm{l}$, if the participant was using glucose-lowering medication, and/or if the participant self-reported to have been diagnosed with diabetes by a health care professional. Among those having diabetes, awareness was defined as self-reported diagnosis of diabetes. Medical treatment was defined as the use of glucose-lowering medication. Among those treated with medication, diabetes control was defined as HbAlc levels $\leq 53 \mathrm{mmol} / \mathrm{mol}$ (7.0\%) [23].

2.4. Covariates. Information on socioeconomic status (level of education) and lifestyle factors (smoking, alcohol use, and physical activity) was obtained by questionnaire. Educational level was based on the highest qualification attained, either in Netherlands or in the country of origin, and it was categorized into four groups: (1) never been to school or elementary schooling only, (2) lower vocational schooling or lower secondary schooling, (3) intermediate vocational schooling or intermediate/secondary schooling, or (4) higher vocational schooling or university. Alcohol intake in the past 12 months (yes/no) and current smoking status (yes/no) were obtained. Habitual physical activity was measured with questions about the time spent on several activities during a normal week in the past few months using the Short Questionnaire to Assess Health-Enhancing Physical Activity (SQUASH) [24]. Participants were categorized as adherent (yes/no) to the Dutch guideline for physical activity when the sum of the number of days per week for each moderate- and highintensity activity lasting at least $30 \mathrm{~min}$ was greater than or equal to five. Weight, height, waist circumference, and hip 


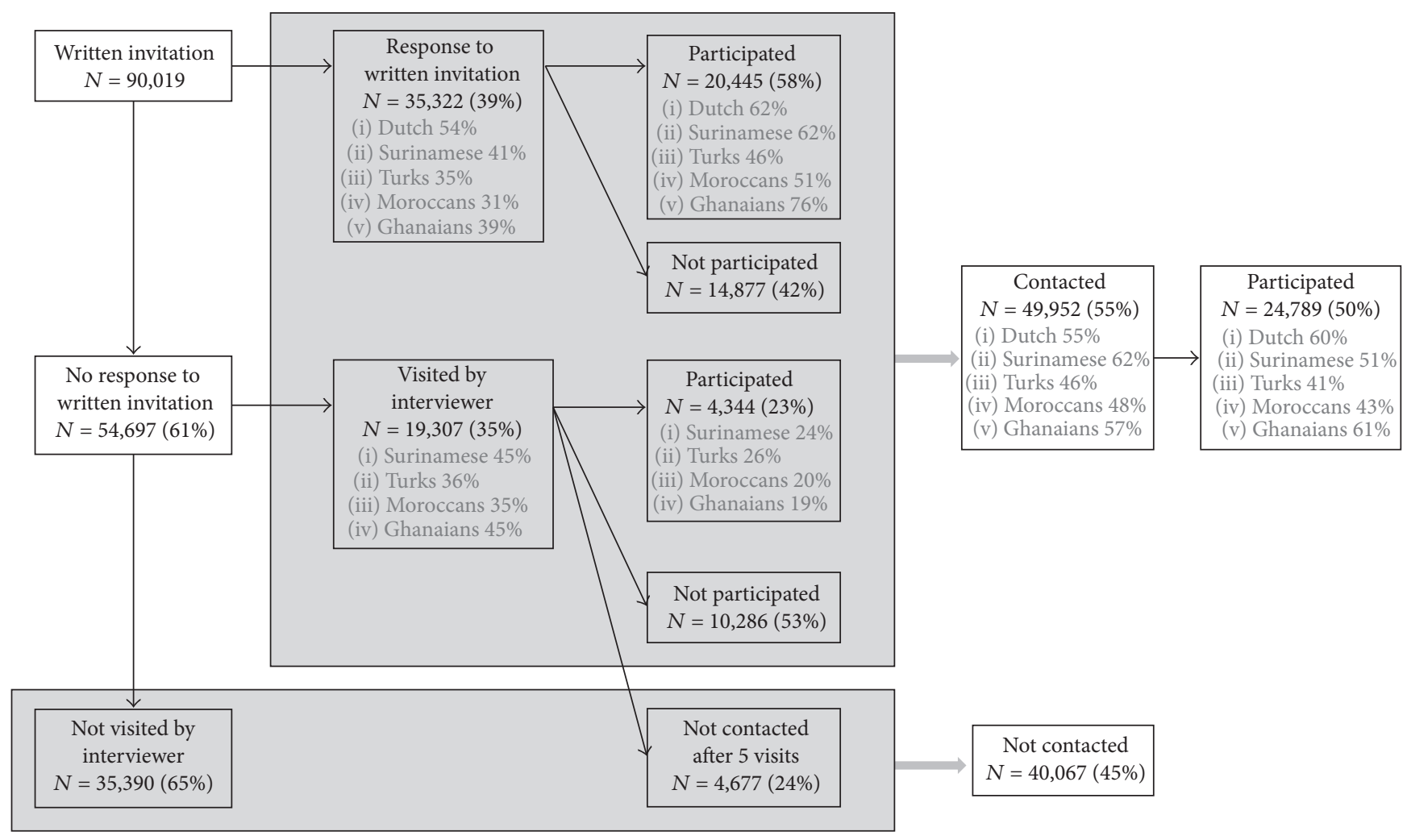

FIGURE 1: Flowchart of recruitment for the HELIUS study.

circumference were measured, and body mass index (BMI) and waist-to-hip ratio (WHR) were calculated, as described before [25].

2.5. Statistical Analyses. Characteristics of men and women in the different ethnic groups were described by means with standard deviations (continuous variables) or percentages (categorical variables). The prevalence of diabetes was calculated, stratified by ethnicity, sex, and 10-year age groups. Then, logistic regression analyses were performed to study ethnic differences in the prevalence of diabetes, adjusted for age and sex. Effect modification by sex was tested, by adding interaction terms to the regression models. Because of significant differences between men and women $(p<0.05)$, all further analyses were performed stratified by sex. We additionally adjusted for well-known risk factors ((abdominal) obesity, health-related behaviors, and education) by adding these variables to the regression models. Finally, we performed logistic regression analyses to study ethnic differences in awareness (among those with diabetes), medical treatment (among those with diabetes as well as among those aware of diabetes only), and control (among those receiving glucoselowering medication), adjusted for age. Statistical analyses were performed using IBM SPSS Statistics version 23 .

\section{Results}

Among both men and women, the Turkish and Moroccan groups were about 5 years younger than the other ethnic groups (Table 1). The Dutch participants were higher educated as compared with all other ethnic minority groups. Large differences in health-related behavior were found between the different ethnic groups. For example, smoking rates were particularly low among Ghanaian participants, whereas alcohol use was particularly low among Moroccan participants. All ethnic minority groups had higher BMI and WHR as compared with the Dutch participants. The unadjusted prevalence of diabetes, as well as known diabetes (self-reported), was higher among all ethnic minority groups as compared with Dutch participants.

In all ethnic groups, the prevalence of diabetes increased with age (Figure 2). The prevalence of diabetes among ethnic minority groups was much higher than that among Dutch participants, and this difference was already apparent and significant at the age of 31-40 years. The prevalence of diabetes among ethnic minority groups within a certain age group was comparable with the prevalence of diabetes among Dutch participants who were about 2 decades younger. For example, prevalence of diabetes in the oldest age group (61-70 years) among Dutch participants was similar to the prevalence among ethnic minority groups who were 41-50 years old (both men and women). Similarly, the prevalence of diabetes in the 51-60-year-old Dutch participants was similar to the prevalence among ethnic minority groups aged 31-40 years.

The ethnic differences in the prevalence of diabetes were also significant after adjustment for age, in both men and women (Table 2). Among men, ethnic minority groups had 
TABLE 1: Characteristics of the study population, by ethnicity and sex.

\begin{tabular}{|c|c|c|c|c|c|c|}
\hline & Dutch & South-Asian Surinamese & African Surinamese & Ghanaian & Turkish & Moroccan \\
\hline \multicolumn{7}{|l|}{ Men } \\
\hline$N$ & 2080 & 1364 & 1598 & 901 & 1624 & 1505 \\
\hline Age (y) & $46.9 \pm 13.8$ & $44.8 \pm 13.6$ & $48.2 \pm 12.9$ & $46.9 \pm 11.5$ & $40.9 \pm 12.1$ & $42.1 \pm 12.8$ \\
\hline \multicolumn{7}{|l|}{ Education (\%) } \\
\hline 1 (lowest) & 3.4 & 13.0 & 6.6 & 15.9 & 24.6 & 25.7 \\
\hline 2 & 13.6 & 32.4 & 40.8 & 45.9 & 30.8 & 21.8 \\
\hline 3 & 23.4 & 30.7 & 34.0 & 29.4 & 28.5 & 33.4 \\
\hline 4 (highest) & 59.6 & 23.9 & 18.6 & 8.9 & 16.0 & 19.1 \\
\hline Current smoking (\% yes) & 26.2 & 39.8 & 42.9 & 7.6 & 41.1 & 26.3 \\
\hline Alcohol (\% yes) & 93.7 & 67.1 & 79.2 & 53.8 & 34.9 & 12.9 \\
\hline Achieving PA norm (\% yes) & 73.0 & 58.0 & 69.0 & 62.5 & 50.0 & 56.1 \\
\hline $\operatorname{BMI}\left(\mathrm{kg} / \mathrm{m}^{2}\right)$ & $25.2 \pm 3.8$ & $25.8 \pm 4.2$ & $26.3 \pm 4.4$ & $26.7 \pm 3.8$ & $27.9 \pm 4.4$ & $26.7 \pm 4.0$ \\
\hline WHR & $0.94 \pm 0.07$ & $0.97 \pm 0.08$ & $0.93 \pm 0.07$ & $0.94 \pm 0.07$ & $0.96 \pm 0.07$ & $0.94 \pm 0.07$ \\
\hline Diabetes $^{\dagger}$ (\% yes) & 5.0 & 21.5 & 11.5 & 14.9 & 11.3 & 12.0 \\
\hline Known diabetes $^{\infty}$ (\% yes) & 3.0 & 17.6 & 8.2 & 12.4 & 8.3 & 9.8 \\
\hline \multicolumn{7}{|l|}{ Women } \\
\hline$N$ & 2461 & 1668 & 2511 & 1422 & 1967 & 2382 \\
\hline Age (y) & $45.6 \pm 14.2$ & $46.1 \pm 13.2$ & $47.8 \pm 12.3$ & $43.4 \pm 10.7$ & $40.0 \pm 12.2$ & $39.4 \pm 12.9$ \\
\hline \multicolumn{7}{|l|}{ Education (\%) } \\
\hline 1 (lowest) & 3.2 & 15.7 & 5.0 & 37.0 & 37.5 & 34.6 \\
\hline 2 & 14.8 & 34.1 & 32.7 & 36.3 & 20.0 & 15.5 \\
\hline 3 & 20.7 & 27.9 & 36.6 & 22.3 & 28.6 & 33.3 \\
\hline 4 (highest) & 61.3 & 22.3 & 25.8 & 4.5 & 14.0 & 16.6 \\
\hline Current smoking (\% yes) & 23.3 & 19.0 & 24.5 & 2.6 & 29.2 & 5.3 \\
\hline Alcohol (\% yes) & 88.8 & 47.4 & 62.1 & 43.5 & 12.7 & 3.9 \\
\hline Achieving PA norm (\% yes) & 77.8 & 49.6 & 56.5 & 47.3 & 35.1 & 40.9 \\
\hline BMI $\left(\mathrm{kg} / \mathrm{m}^{2}\right)$ & $24.4 \pm 4.5$ & $26.7 \pm 5.3$ & $28.8 \pm 5.9$ & $29.6 \pm 5.3$ & $29.1 \pm 6.6$ & $28.1 \pm 5.8$ \\
\hline WHR & $0.84 \pm 0.08$ & $0.90 \pm 0.08$ & $0.88 \pm 0.08$ & $0.88 \pm 0.08$ & $0.87 \pm 0.09$ & $0.86 \pm 0.09$ \\
\hline Diabetes $^{\dagger}$ (\% yes) & 2.4 & 17.7 & 12.1 & 9.6 & 9.3 & 10.8 \\
\hline Known diabetes $^{\infty}$ (\% yes) & 1.9 & 16.0 & 10.5 & 7.6 & 8.1 & 9.3 \\
\hline
\end{tabular}

Data are mean with standard deviation or percentages. BMI = body mass index. WHR = waist-to-hip ratio.

${ }^{\dagger}$ Diabetes based on self-report, fasting glucose $\geq 7 \mathrm{mmol} / \mathrm{l}$, and/or use of glucose-lowering medication.

${ }^{\infty}$ Diabetes based on self-report.

TABLE 2: Ethnic differences in the prevalence of diabetes ${ }^{\dagger}$ by sex, adjusted for age.

\begin{tabular}{|c|c|c|c|c|c|c|c|c|}
\hline & \multicolumn{4}{|c|}{ Men } & \multicolumn{4}{|c|}{ Women } \\
\hline & $n / N$ & $\%$ & OR & $95 \% \mathrm{CI}$ & $n / N$ & $\%$ & OR & $95 \% \mathrm{CI}$ \\
\hline Dutch & $105 / 2080$ & 5.0 & 1.0 (ref) & & $58 / 2461$ & 2.4 & 1.0 (ref) & \\
\hline South-Asian Surinamese & $293 / 1364$ & 21.5 & 8.0 & $6.3-10.4$ & $296 / 1668$ & 17.7 & 12.2 & $9.0-16.5$ \\
\hline African Surinamese & $183 / 1598$ & 11.5 & 2.6 & $2.0-3.4$ & $303 / 2511$ & 12.1 & 6.5 & $4.8-8.7$ \\
\hline Ghanaian & $134 / 901$ & 14.9 & 4.6 & $3.5-6.1$ & $136 / 1422$ & 9.6 & 8.9 & $6.4-12.4$ \\
\hline Turkish & $183 / 1624$ & 11.3 & 5.3 & $4.1-7.0$ & $183 / 1967$ & 9.3 & 10.5 & $7.6-14.5$ \\
\hline Moroccan & $181 / 1505$ & 12.0 & 4.8 & $3.7-6.3$ & $258 / 2124$ & 10.8 & 12.3 & $9.0-16.7$ \\
\hline
\end{tabular}

${ }^{\dagger}$ Diabetes based on self-report, fasting glucose $\geq 7 \mathrm{mmol} / \mathrm{l}$, and/or use of glucose-lowering medication.

3 to 8 times higher odds to have diabetes as compared with Dutch participants, and ethnic minority women had 6 to 12 times higher odds compared to Dutch women. Even after adjustment for conventional risk factors for diabetes (i.e., BMI, WHR, alcohol intake, smoking, physical activity, and educational level), ethnic minority groups had 3 to 5 times higher odds to have diabetes as compared with Dutch participants (data not shown).

Among all ethnic minority groups, the odds of being aware of diabetes were higher as compared with Dutch participants, though not statistically significant for Ghanaian women (Table 3 ). The awareness of diabetes was $70-80 \%$ 


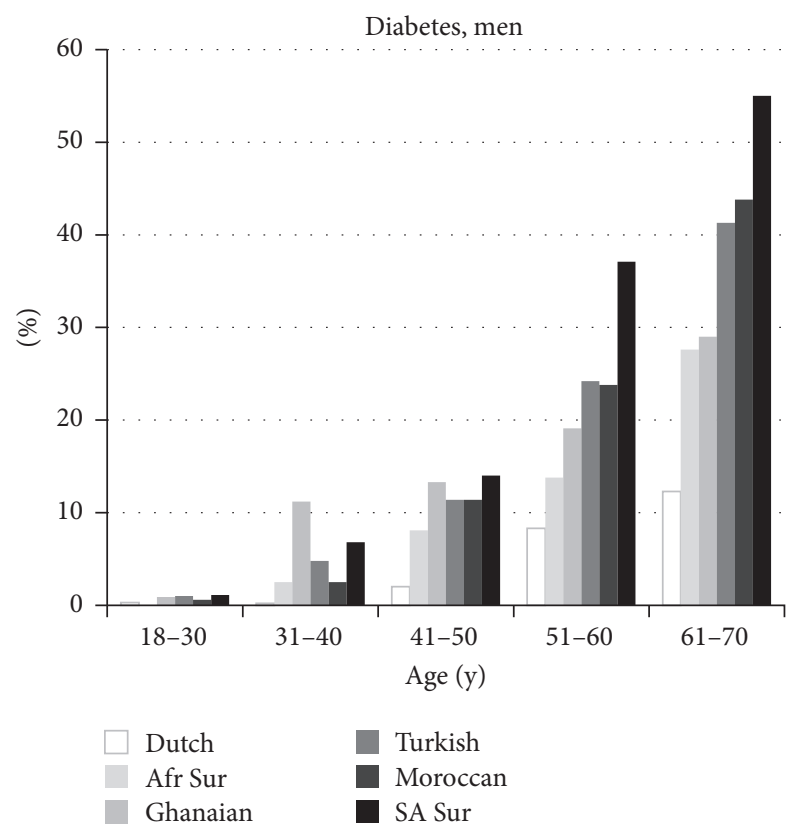

(a)

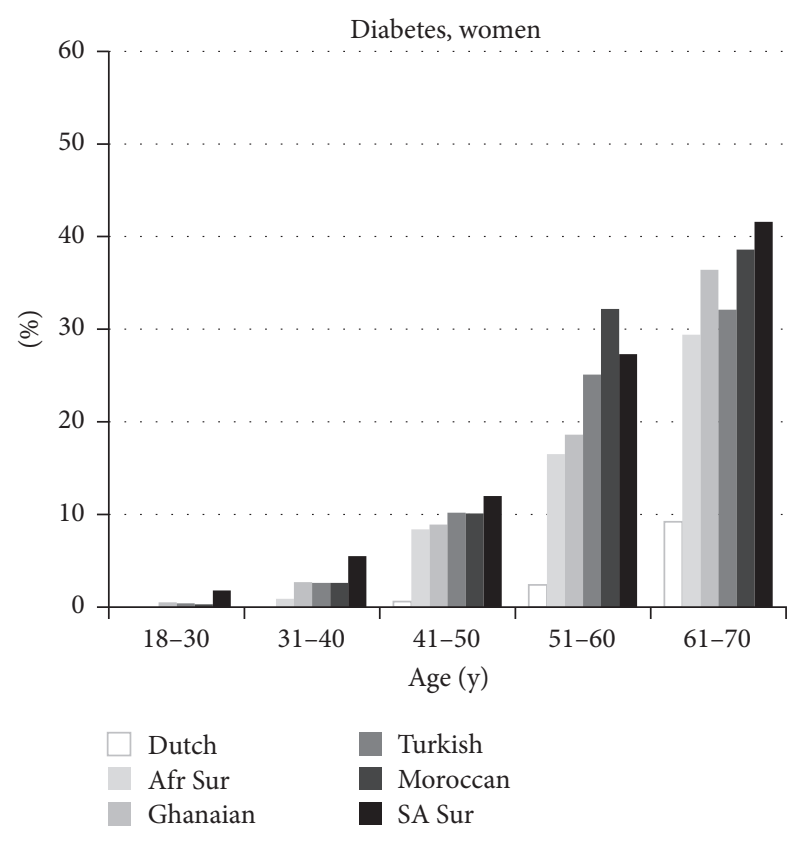

(b)

Figure 2: (a) The prevalence of diabetes by ethnicity, sex, and age groups (men). Afr Sur, African Surinamese; SA Sur, South-Asian Surinamese. (b) The prevalence of diabetes by ethnicity, sex, and age groups (women). Afr Sur, African Surinamese; SA Sur, South-Asian Surinamese.

among ethnic minority men, compared with $60 \%$ among Dutch men. Among women, the awareness was $80-90 \%$. The odds of receiving medical treatment for diabetes were also higher in all ethnic minority groups as compared with Dutch participants. However, when restricting the medical treatment analyses to those aware of their diabetes, the odds of receiving medical treatment did not significantly differ between ethnic groups, except that African Surinamese and South-Asian Surinamese men had significantly higher odds of receiving medical treatment as compared with Dutch participants.

Among those treated with glucose-lowering medication, all ethnic minority men had significantly lower odds of controlled HbAlc levels as compared with Dutch men (control rates of $37.4-45.6 \%$ versus $67.4 \%$, resp., Table 3 ). This could not be explained by the type of diabetes medication (oral or insulin), as the percentage of those using oral medication/insulin did not substantially differ between the Dutch and the other ethnic groups (94\% using oral and 23\% using insulin). Also, the age of onset of diabetes and diabetes duration among Dutch men were similar to the other ethnic groups (data not shown). In women, there were no significant ethnic differences in glycemic control (control rates ranging from 38.2 to $52.9 \%$ ), while Dutch women relatively more often used insulin medication (35\% versus $22-28 \%$ in the ethnic minority groups) and had the highest age of onset of diabetes and the longest diabetes duration compared with the other ethnic groups (data not shown).

\section{Discussion}

Already at young age, the prevalence of type 2 diabetes was higher among all ethnic minority groups as compared with the Dutch host population. Our results suggest a 20 -year earlier onset of diabetes among the ethnic minority groups than that among the Dutch participants. Awareness and treatment rates of diabetes were significantly higher among ethnic minority groups compared with Dutch participants. If aware, all ethnic groups had similar odds of receiving medical treatment. Among those treated with glucose-lowering medication, despite high levels of awareness, only 37 to $53 \%$ had their HbAlc levels on target, except for Dutch men (67\%). In women there were no significant ethnic differences in glycemic control.

Our finding of a higher prevalence of diabetes among ethnic minority populations as compared to the host population, even after adjustment for conventional diabetes risk factors, was consistent with several previous studies [8-13]. Our results also confirm previous smaller studies [11, 15, $17,19]$ that suggested that diabetes starts to develop at a much younger age in ethnic minority groups as compared to Dutch participants. These findings suggest that case finding for diabetes should possibly start at younger age among these ethnic minority groups than that among the general population. The ADA suggests screening from the age of 45 years in overweight individual and suggests screening before the age of 45 years in overweight individuals who are of "high risk race/ethnicity" [23]. However, which ethnic groups, or from what age, is not specified. Guidelines in the UK suggest screening from the age of 25 years among South-Asians only [26]. Current Dutch general practice standards also suggest screening from the age of 45 years, and from the age of 35 in South-Asians, if a risk factor for diabetes (e.g., overweight or family history of diabetes) is present. Because the prevalence of diabetes among ethnic minority groups at age 31-40 years 


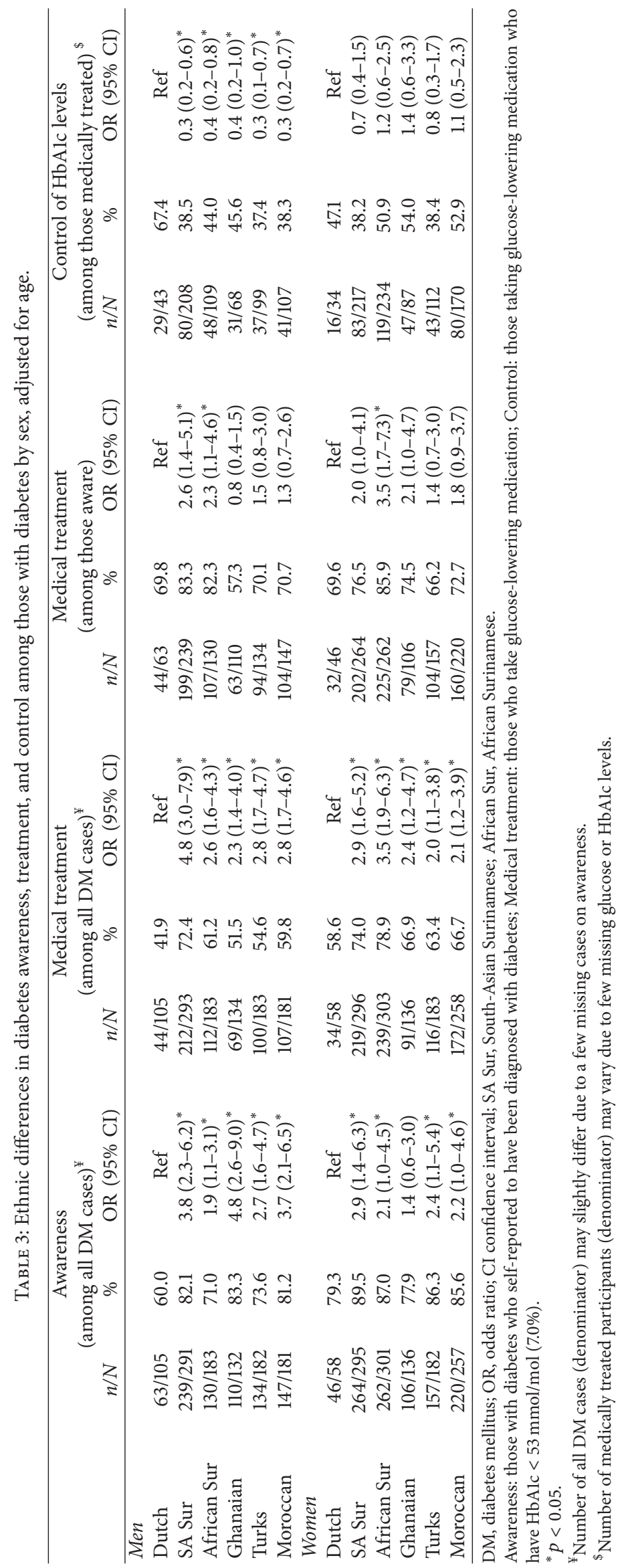


is similar to or even higher than the prevalence among Dutch participants at the suggested screening age of 45 years (41-50 years), our results suggest that the age limit should be lowered for African, South-Asian, Turkish, and Moroccan minority groups, rather than for South-Asians only.

Consistent with previous European studies [14-16], we did not find a lower diabetes awareness among ethnic minority groups as compared with the Dutch host population. US studies reported a lower awareness among ethnic minority groups $[5,6]$ and suggested this to be due to a lower percentage of ethnic minority people with health insurance, resulting in lower access to health care [6]. In Netherlands, a standard health insurance is legally obliged, which may explain the comparable or even higher awareness among the ethnic minority groups compared with Dutch participants in our study. These findings are consistent with recent findings on a higher hypertension awareness among ethnic minority groups compared with the Dutch host population in Netherlands [27]. Some studies have also suggested that ethnic minority groups are less likely to be prescribed lipid lowering and antihypertensive drugs compared with the host population $[19,28]$. However, we found that levels of medical treatment with glucose-lowering agents were similar, or even higher, among ethnic minority groups. This, again, is probably due to the equal access to health care in Netherlands and is also consistent with recent findings on similar treatment rates among different ethnic groups in Netherlands [27].

Ethnic differences in glycemic control have previously been found in both US and UK studies, with lower control among ethnic minority groups $[5,17-19,29]$. Ethnic differences in control have been suggested to be due to poorer patient concordance (health literacy, poorer care standards), poorer quality of care, or lower response to diabetic agents among ethnic minority groups. In our study, despite equal access to health care, we found poorer glycemic control among ethnic minority men compared with Dutch men, which could not be contributed to differences in type of medication, diabetes duration, or age of onset of diabetes. Among women, there were no ethnic differences in glycemic control, and the level of control among women was similar to that of ethnic minority men. It is unclear why Dutch men showed a much higher level of glycemic control compared to all the other ethnic groups and compared with women. For hypertension control, control rates among men were consistently lower or similar when compared to women, in all ethnic groups (including Dutch) [27]. For glycemic control we observe the same gender difference, except for Dutch men. Regardless of ethnicity, however, the majority of medically treated patients did not have their HbAlc levels on target, suggesting that efforts to improve glycemic control should not only include ethnic minority groups. Noncompliance to treatment, lack of understanding of the disease, type of medication, or poor quality of health care professionals could all be involved in explaining these low glycemic control levels and require further investigation.

A limitation of our study is that fasting glucose was only measured on one single occasion. Therefore, there may have been some misclassification, and the prevalence of newly detected diabetes may have been somewhat overestimated, thereby affecting the estimation of awareness levels. However, this applies to all ethnic groups and if measured twice this would likely have led to even higher estimated awareness. Another limitation of our study is that we only studied treatment by glucose-lowering medication, whereas lifestyle interventions (either alone or in combination with medical treatment) may also help to obtain glycemic control. Of the different possible interventions, however, one would expect that medical treatment would have the highest impact on lowering glucose levels, whereas even in this group glycemic control was disappointing.

\section{Conclusion}

South-Asian, African, Turkish, and Moroccan ethnic minority groups had a much higher prevalence of type 2 diabetes compared with the Dutch host population. The high prevalence among these ethnic minority groups already occurred at young age, suggesting that the age limit for case finding should possibly be lowered for all these ethnic groups. Given the high prevalence of diabetes among young ethnic minority groups and the low levels of glycemic control in general, adequate detection and treatment deserve increased attention, particularly among ethnic minority groups. Further research should focus on the causes of poor glycemic control among those using glucose-lowering medication.

\section{Competing Interests}

The authors declare that there is no conflict of interests regarding the publication of this paper.

\section{Acknowledgments}

The HELIUS study is conducted by the Academic Medical Center Amsterdam and the Public Health Service of Amsterdam. Both organizations provided core support for HELIUS. The HELIUS study is also funded by the Dutch Heart Foundation (Grant no. 2010T084), the Netherlands Organization for Health Research and Development (ZonMw) (Grant no. 200500003), and the European Union (FP-7) (Grant no. 278901). The authors are most grateful to the participants of the HELIUS study and the management team, research nurses, interviewers, research assistants, and other staff who have taken part in gathering the data of this study.

\section{References}

[1] R. C. Turner, C. A. Cull, V. Frighi, and R. R. Holman, "Glycemic control with diet, sulfonylurea, metformin, or insulin in patients with type 2 diabetes mellitus. Progressive requirement for multiple therapies (UKPDS 49)," Journal of the American Medical Association, vol. 281, no. 21, pp. 2005-2012, 1999.

[2] R. R. Holman, S. K. Paul, M. A. Bethel, D. R. Matthews, and H. A. W. Neil, "10-Year follow-up of intensive glucose control in type 2 diabetes," New England Journal of Medicine, vol. 359, no. 15, pp. 1577-1589, 2008.

[3] J. Tuomilehto, J. Lindström, J. G. Eriksson et al., "Prevention of type 2 diabetes mellitus by changes in lifestyle among subjects with impaired glucose tolerance," The New England Journal of Medicine, vol. 344, no. 18, pp. 1343-1350, 2001. 
[4] C. L. Gillies, K. R. Abrams, P. C. Lambert et al., "Pharmacological and lifestyle interventions to prevent or delay type 2 diabetes in people with impaired glucose tolerance: systematic review and meta-analysis," British Medical Journal, vol. 334, no. 7588, pp. 299-302, 2007.

[5] E. Selvin, C. M. Parrinello, D. B. Sacks, and J. Coresh, "Trends in prevalence and control of diabetes in the United States, 19881994 and 1999-2010," Annals of Internal Medicine, vol. 160, no. 8, pp. 517-525, 2014.

[6] A. Menke, S. Casagrande, L. Geiss, and C. C. Cowie, "Prevalence of and trends in diabetes among adults in the United States, 1988-2012," The Journal of the American Medical Association, vol. 314, no. 10, pp. 1021-1029, 2015.

[7] I. S. Okosun and G. E. A. Dever, "Abdominal obesity and ethnic differences in diabetes awareness, treatment, and glycemic control," Obesity Research, vol. 10, no. 12, pp. 1241-1250, 2002.

[8] H. Dijkshoorn, D. G. Uitenbroek, and B. J. C. Middelkoop, "Prevalence of diabetes mellitus and cardiovascular disease among immigrants from Turkey and Morocco and the indigenous Dutch population," Nederlands Tijdschrift voor Geneeskunde, vol. 147, no. 28, pp. 1362-1366, 2003.

[9] P. E. Wändell, K. H. Steiner, and S. E. Johansson, "Diabetes mellitus in Turkish immigrants in Sweden," Diabetes and Metabolism, vol. 29, no. 4, pp. 435-439, 2003.

[10] J. K. Kristensen, J. F. Bak, I. Wittrup, and T. Lauritzen, "Diabetes prevalence and quality of diabetes care among Lebanese or Turkish immigrants compared to a native Danish population," Primary Care Diabetes, vol. 1, no. 3, pp. 159-165, 2007.

[11] J. K. Ujcic-Voortman, M. T. Schram, M. A. Jacobs-van der Bruggen, A. P. Verhoeff, and C. A. Baan, "Diabetes prevalence and risk factors among ethnic minorities," European Journal of Public Health, vol. 19, no. 5, pp. 511-515, 2009.

[12] J. K. Ujcic-Voortman, C. A. Baan, J. C. Seidell, and A. P. Verhoeff, "Obesity and cardiovascular disease risk among Turkish and Moroccan migrant groups in Europe: a systematic review," Obesity Reviews, vol. 13, no. 1, pp. 2-16, 2012.

[13] K. A. C. Meeks, D. Freitas-Da-Silva, A. Adeyemo et al., "Disparities in type 2 diabetes prevalence among ethnic minority groups resident in Europe: a systematic review and meta-analysis," Internal and Emergency Medicine, vol. 11, no. 3, pp. 327-340, 2015.

[14] L. Riste, F. Khan, and K. Cruickshank, "High prevalence of type 2 diabetes in all ethnic groups, including europeans, in a British inner city: relative poverty, history, inactivity, or 21st century Europe?" Diabetes Care, vol. 24, no. 8, pp. 1377-1383, 2001.

[15] N. R. Bindraban, I. G. M. Van Valkengoed, G. Mairuhu et al., "Prevalence of diabetes mellitus and the performance of a risk score among Hindustani Surinamese, African Surinamese and ethnic Dutch: a cross-sectional population-based study," BMC Public Health, vol. 8, article no. 271, 2008.

[16] K. A. C. Meeks, K. Stronks, E. J. A. J. Beune et al., "Prevalence of type 2 diabetes and its association with measures of body composition among African residents in the Netherlands-the HELIUS study," Diabetes Research and Clinical Practice, vol. 110, pp. 137-146, 2015.

[17] P. H. Negandhi, N. Ghouri, H. M. Colhoun et al., "Ethnic differences in glycaemic control in people with type 2 diabetes mellitus living in Scotland," PLoS ONE, vol. 8, no. 12, Article ID e83292, 2013.

[18] M. A. Soljak, A. Majeed, J. Eliahoo, and A. Dornhorst, "Ethnic inequalities in the treatment and outcome of diabetes in three
English Primary Care Trusts," International Journal for Equity in Health, vol. 6, article 8, 2007.

[19] B. Mukhopadhyay, N. G. Forouhi, B. M. Fisher, C. M. Kesson, and N. Sattar, "A comparison of glycaemic and metabolic control over time among South Asian and European patients with Type 2 diabetes: results from follow-up in a routine diabetes clinic," Diabetic Medicine, vol. 23, no. 1, pp. 94-98, 2006.

[20] K. Stronks, M. B. Snijder, R. J. Peters, M. Prins, A. H. Schene, and A. H. Zwinderman, "Unravelling the impact of ethnicity on health in Europe: the HELIUS study," BMC Public Health, vol. 13, no. 1, article 402, 2013.

[21] K. Stronks, I. Kulu-Glasgow, and C. Agyemang, "The utility of 'country of birth' for the classification of ethnic groups in health research: the Dutch experience," Ethnicity \& Health, vol. 14, no. 3, pp. 255-269, 2009.

[22] WHO Collaborating Centre for Drug Statistics Methodology, Guidelines for ATC Classification and DDD Assignment 2014, WHO Collaborating Centre for Drug Statistics Methodology, Oslo, Norway, 2013.

[23] American Diabetes A, "Standards of medical care in diabetes2013," Diabetes Care, vol. 36, supplement 1, pp. S11-S66, 2013.

[24] G. C. W. Wendel-Vos, A. J. Schuit, W. H. M. Saris, and D. Kromhout, "Reproducibility and relative validity of the short questionnaire to assess health-enhancing physical activity," Journal of Clinical Epidemiology, vol. 56, no. 12, pp. 1163-1169, 2003.

[25] M. B. Snijder, K. Stronks, C. Agyemang, W. B. Busschers, R. J. Peters, and B.-J. H. van den Born, "Ethnic differences in arterial stiffness the Helius study," International Journal of Cardiology, vol. 191, pp. 28-33, 2015.

[26] National Institute for Health and Care Excellence (NICE), Public Health Guideline. Type 2 Diabetes: Prevention in People at High Risk, National Institute for Health and Care Excellence (NICE), 2012.

[27] C. Agyemang, S. Kieft, M. B. Snijder et al., "Hypertension control in a large multi-ethnic cohort in Amsterdam, The Netherlands: The HELIUS study," International Journal of Cardiology, vol. 183, Article ID 19622, pp. 180-189, 2015.

[28] M. G. Patel, D. J. Wright, P. S. Gill, D. Jerwood, J. Silcock, and H. Chrystyn, "Prescribing of lipid lowering drugs to South Asian patients: Ecological Study," British Medical Journal, vol. 325, pp. 25-26, 2002.

[29] M. I. Harris, R. C. Eastman, C. C. Cowie, K. M. Flegal, and M. S. Eberhardt, "Racial and ethnic differences in glycemic control of adults with type 2 diabetes," Diabetes Care, vol. 22, no. 3, pp. 403-408, 1999. 


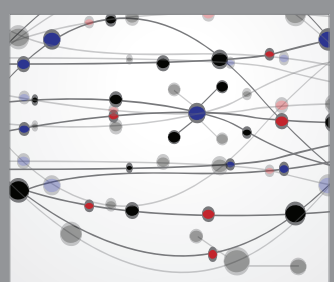

The Scientific World Journal
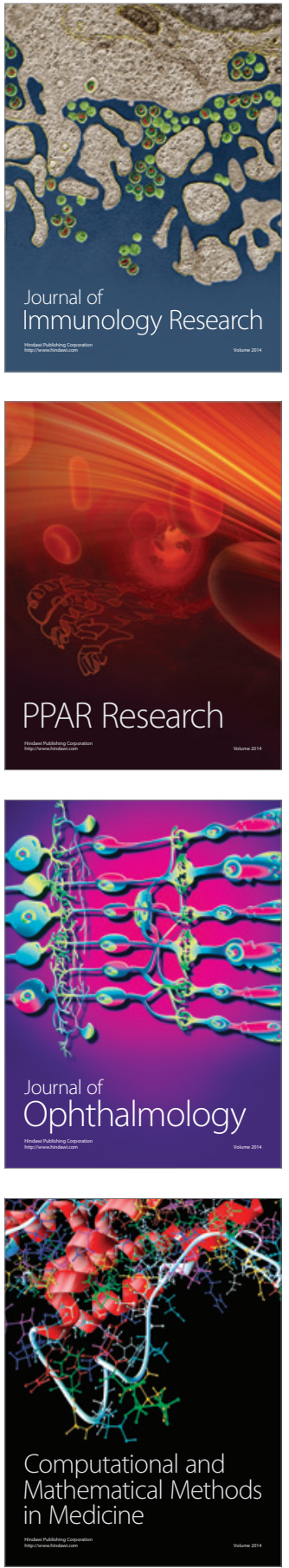

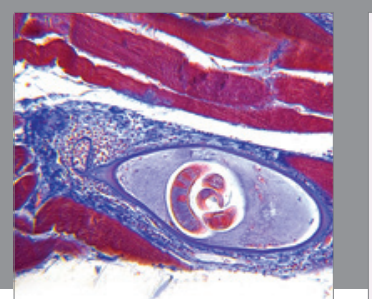

Gastroenterology Research and Practice
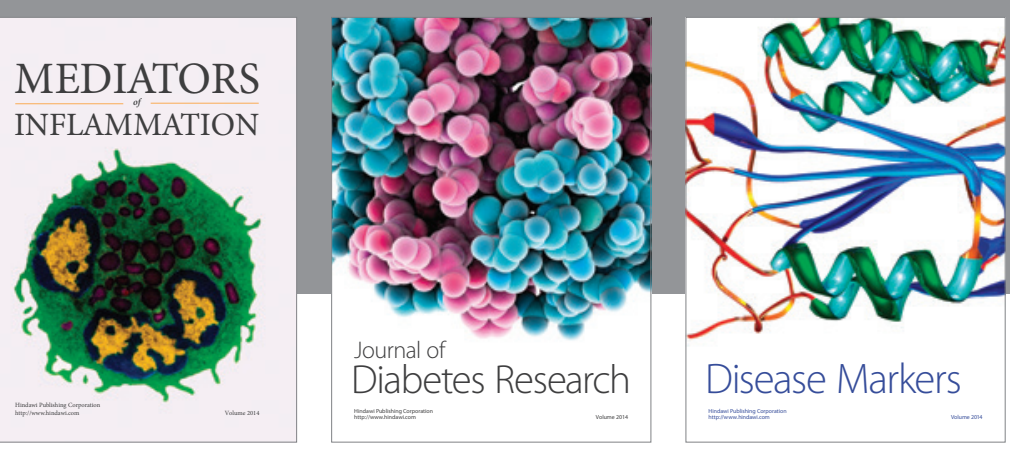

Disease Markers

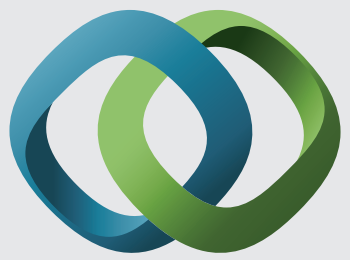

\section{Hindawi}

Submit your manuscripts at

https://www.hindawi.com
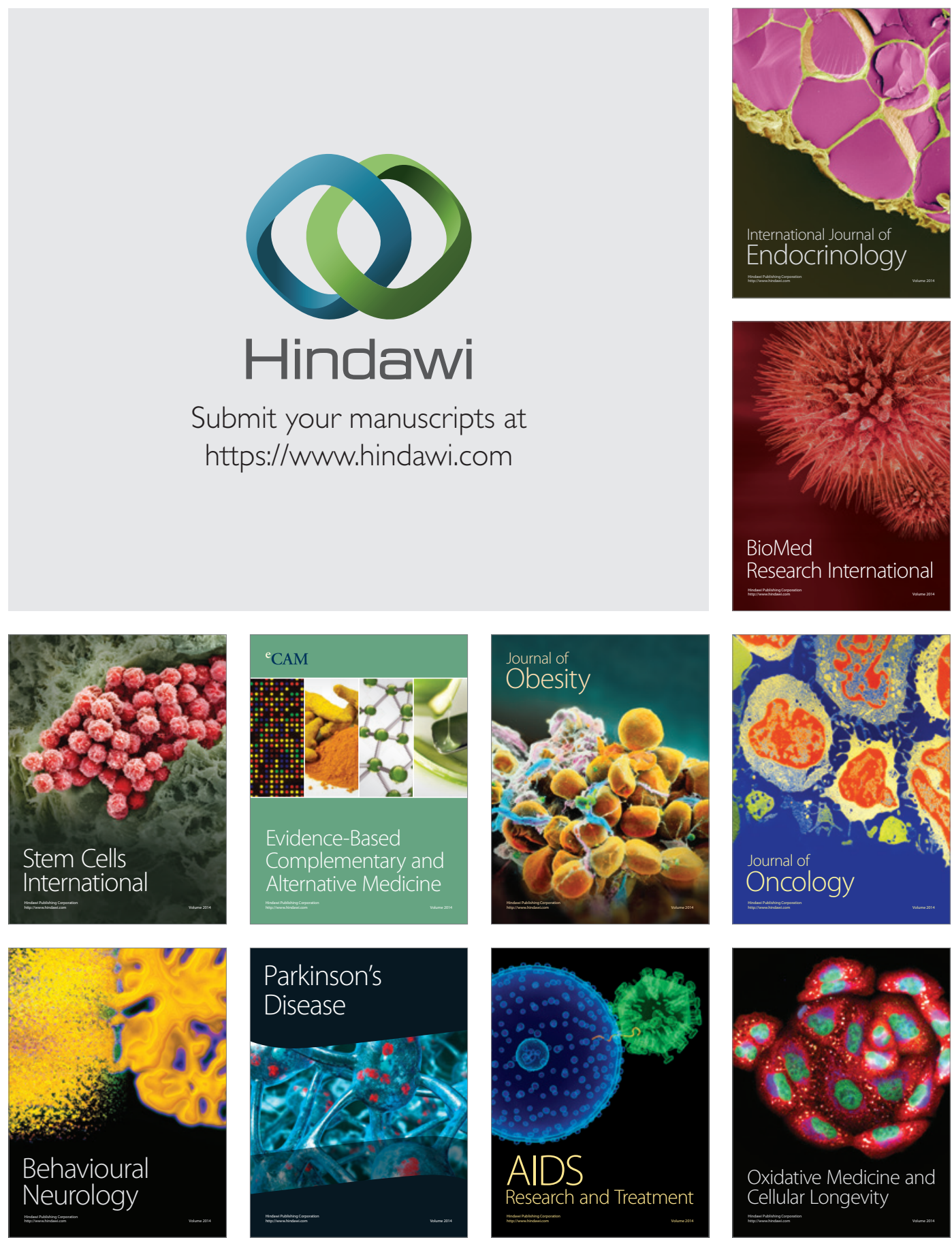